\title{
0 efeito trabalhador adicional para filhos no Brasil
}

\author{
Elzira Lúcia de Oliveira* \\ Eduardo Gonçalves Rios-Neto* \\ Ana Maria Hermeto Camilo de Oliveira ${ }^{* * *}$
}

O objetivo deste trabalho é testar a hipótese da existência do efeito trabalhador adicional para filhos no Brasil, procurando identificar se a situação de desemprego do chefe de família fará com que algum membro da família, cuja condição seja filho, transite para a População Economicamente Ativa - PEA. A base de dados utilizada foi a Pesquisa Mensal de Emprego PME realizada pelo IBGE, que permite a construção de painéis para análise longitudinal de dados. A hipótese foi testada para pelo menos um filho com idade de 10 a 18 anos, entre 2002 e 2013, para as regiões metropolitanas de Belo Horizonte, Salvador, Porto Alegre, São Paulo, Rio de Janeiro e Recife, que compõem a área de abrangência da PME. Este trabalho admite a existência de diferencial por sexo do chefe para o efeito trabalhador adicional de filhos de 10 a 18 anos. Os resultados mostraram haver um efeito positivo maior para chefes homens do que para chefes mulheres, sendo que a variável de transição do filho para a atividade não apresentou significância estatística que permitisse assumir a existência do efeito. Corroborou-se a hipótese da existência de diferencial por sexo, contudo no sentido oposto ao da hipótese assumida.

Palavras-chave: Efeito trabalhador adicional. Desemprego. Mercado de trabalho.

\footnotetext{
*Universidade Federal Fluminense - UFF Campos, Campos dos Goytacazes-RJ, Brasil (elziralucia@globo.com).

** Centro de Planejamento Regional - Cedeplar da Universidade Federal de Minas Gerais - UFMG, Belo Horizonte-MG, Brasil (eduardo@cedeplar.ufmg.br).

${ }^{* * *}$ Centro de Planejamento Regional - Cedeplar da Universidade Federal de Minas Gerais - UFMG, Belo Horizonte-MG, Brasil (ahermeto@cedeplar.ufmg.br).
} 


\section{Introdução}

A questão da entrada de crianças e adolescentes na força de trabalho é discutida a partir de várias perspectivas teóricas. Em geral, os estudos investigam os determinantes da alocação do tempo desses indivíduos entre a escola e o trabalho por meio de estratégias metodológicas diversas (CORSEUIL et. al., 2000; LEME; WAJNMAN, 2000; KASSOUF, 2000). Uma abordagem teórica que tem sido pouco explorada na literatura nacional e na internacional é o efeito trabalhador adicional para crianças e adolescentes, ou seja, a entrada dos filhos, em particular crianças e adolescentes, no mercado de trabalho em decorrência do desemprego do chefe de família.

O efeito trabalhador adicional consiste no impacto do desemprego do chefe de família sobre a oferta de trabalho de outros membros. Tanto na literatura americana quanto na europeia, a preocupação maior tem sido com a entrada das esposas no mercado de trabalho em decorrência do desemprego do marido. Na literatura brasileira, o foco também não é diferente. Os poucos estudos identificados, a exemplo de Fernandes e Felício (2002), buscam verificar a existência deste efeito para esposas, particularmente, no mercado de trabalho metropolitano brasileiro, devido à especificidade da base de dados comumente utilizada: a Pesquisa Mensal de Emprego - PME.

O objetivo deste trabalho é testar a hipótese da existência do efeito trabalhador adicional para filhos no Brasil. Em outras palavras, procura-se verificar se a situação de desemprego do chefe de família faz com que algum membro da família, cuja condição seja filho, ingresse na População Economicamente Ativa - PEA. A base de dados utilizada foi a Pesquisa Mensal de Emprego - PME, realizada pelo IBGE, que permite a construção de painéis para análise longitudinal de dados. Assim, será testada a hipótese para pelo menos um filho com idade de 10 a 18 anos, entre 2002 e 2013, para as regiões metropolitanas de Belo Horizonte, Salvador, Porto Alegre, São Paulo, Rio de Janeiro e Recife, que compõem a área de abrangência da PME.

Este trabalho inova ao avaliar o impacto do desemprego dos pais sobre a participação dos filhos na força de trabalho e contribui para o aumento de estudos sobre esse tema na literatura latino-americana. Assim, a pergunta para a qual se busca a resposta é: existe efeito trabalhador adicional para filhos com idade de 10 a 18 anos no Brasil metropolitano entre 2002 e 2013? A hipótese que se coloca é que há um efeito trabalhador adicional positivo para filhos neste intervalo etário no Brasil.

Como hipótese adicional deste trabalho, admite-se a existência de diferencial por sexo do chefe de família para o efeito trabalhador adicional de filhos de 10 a 18 anos. Acredita-se que, na maior parte dos casos, quando o chefe de família é do sexo masculino, é pressuposta uma estrutura familiar composta de, pelo menos, um chefe e uma esposa. Em famílias chefiadas por mulheres, geralmente, verificam-se outras relações de parentesco, que não os de cônjuge. Sendo isso verdade, pode-se supor que, nas famílias chefiadas por homens, o primeiro candidato natural para substituir o chefe desempregado no mercado de trabalho seria a esposa. O segundo candidato natural seria o filho mais velho ou qualquer um dos 
filhos aptos ao trabalho, no caso de baixa produtividade da esposa no mercado de trabalho. Já no caso de famílias chefiadas por mulheres, o primeiro candidato natural para substituir o chefe desempregado no mercado de trabalho seria o filho. Então, supor que o efeito trabaIhador adicional para filhos de mulheres chefes de família seja maior do que para filhos de homens chefes é uma hipótese plausível, como mostram as Figuras 1 e 2.

FIGURA 1

Representação esquemática da hipótese do efeito trabalhador adicional para filhos de mulheres chefes de família

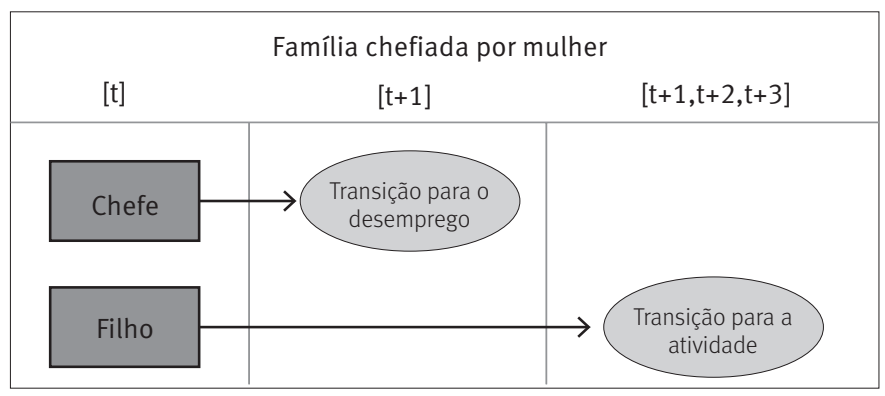

FIGURA 2

Representação esquemática da hipótese do efeito trabalhador adicional para filhos de homens chefes de família

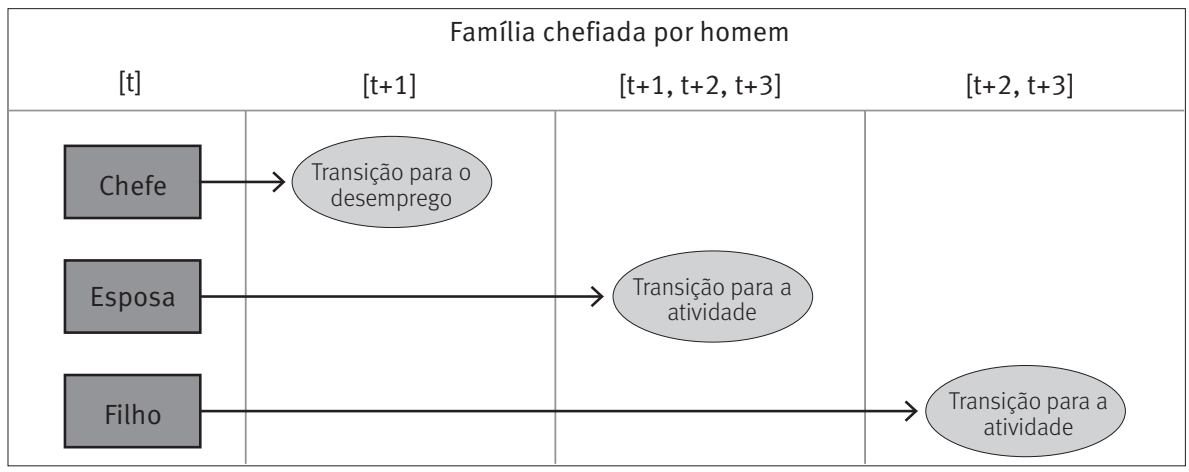

Sendo [t] o mês no qual se inicia a observação do evento, ou seja, quando o chefe de família se encontra ocupado e o filho inativo, os meses subsequentes referem-se à transição do chefe para o desemprego em $\mathrm{t}+1$ e à transição para a atividade do filho em [t+1, t+2 e t+3].

Espera-se, neste trabalho, encontrar um efeito positivo e maior do que o verificado por Oliveira (2005) para filhos mais velhos, uma vez que o fato de se testar a transição para a atividade apenas para o filho mais velho ignorava a probabilidade de qualquer outro filho, inativo em t, transitar para atividade em virtude do desemprego do chefe de família. No caso particular deste artigo, eliminou-se a restrição sobre o filho mais velho e restringiu-se o intervalo etário de observação para 10 a 18 anos, pois admite-se que os filhos com idade superior a 18 anos apresentam maior probabilidade de ingressar na força de trabalho, independentemente do desemprego dos pais. 
Além desta introdução, o artigo traz a seção de antecedentes teóricos, na qual é realizada uma breve discussão da literatura. Também são especificadas a metodologia e a fonte de dados e comentadas as estimativas em relação ao desenho amostral. Posteriormente são analisados e discutidos os resultados encontrados e, na última seção, apresentam-se as considerações finais à luz dos resultados obtidos.

\section{Antecedentes teóricos}

De acordo com Ehrenberg e Smith (2000), mudanças na produtividade de um dos cônjuges em casa ou no mercado de trabalho podem afetar a decisão básica de oferta de mão de obra da família. Como a recessão não atinge a produtividade doméstica, é provável que a família considere que o cônjuge se envolva nas atividades domésticas. Se a esposa antes alocava todo o seu tempo na atividade doméstica, ela não tem sua produtividade no mercado afetada. Assim, a fim de tentar manter o nível anterior de utilidade da família, ela deve substituir o cônjuge nas atividades no mercado, e ele substituí-la nas atividades domésticas enquanto durar a recessão. Se, por um lado, o cônjuge que alocava o seu tempo em atividades no mercado continua a fazer parte da força de trabalho na situação de desempregado, por outro, a partir do momento em que a esposa busca um posto de trabalho no mercado, ela se torna um membro adicional da força de trabalho. Então, em face da queda da renda familiar, o número de membros da família que buscam trabalho no mercado pode aumentar, o que pressiona o mercado de trabalho, elevando a taxa de desemprego e exercendo uma pressão descendente sobre os salários.

Como o salário esperado declina em presença de recessão, seja pelo excesso da oferta de mão de obra sobre a demanda seja pela menor probabilidade de se obter um emprego durante uma recessão, o custo de oportunidade de investir na procura de trabalho vis-à-vis ficar em casa torna-se alto, induzindo os indivíduos a se retirarem da força de trabalho. A redução da força de trabalho pelo desencorajamento tende a agir contra o efeito trabalhador adicional. A pressão sobre os salários e sobre a taxa de desemprego será dependente de qual desses efeitos predomina no mercado (os desencorajados ou os trabalhadores adicionais). À medida que a mulher tende a fazer parte da força de trabalho de uma forma constante, o efeito trabalhador adicional estará confinado aos filhos, possivelmente àqueles mais velhos (EHRENBERG; SMITH, 2000).

Congregado et al. (2011) investigam qual dos efeitos - desencorajamento ou trabalhador adicional - predominou sobre a taxa de desemprego da Espanha a partir da crise financeira internacional de 2008. Os autores assumem que esses efeitos são ambíguos por se tratar de um relacionamento time-varying. Eles citam Benati (2001), Darby et al. (2001) e Altavilla et al. (2005) como alguns exemplos de autores que têm testado a hipótese da existência de um relacionamento de longo prazo não linear entre a taxa de participação e a de desemprego, levando em consideração a possível existência de diferenças no relacionamento por sexo e idade como um meio de confirmar a robustez das estimativas. Os resultados mostraram que o efeito trabalhador adicional prevalece sobre o efeito desencorajamento até o limite 
superior de 11,7\% da taxa de desemprego. Acima deste limite, os dois efeitos se anulam mutuamente e a taxa de participação não é influenciada pela piora das condições econômicas.

Em grande parte, os estudos do efeito do trabalhador adicional enfocam a entrada da esposa no mercado de trabalho em face de uma situação do desemprego do marido (STEPHEN, 2001; SPLETZER, 1995; DORIS, 1999; PRIETO-RODRÍGUEZ; RODRÍGUEZ-GUTIÉRREZ, 2000; GRUBER; CULLEN, 1996). A existência do efeito trabalhador adicional varia muito entre famílias e entre países. A literatura americana relata a estimação de um efeito trabalhador adicional fraco para os Estados Unidos, ao passo que, na literatura europeia, o efeito é praticamente ausente. Na literatura recente, particularmente em Doris (1999), as explicações para a ausência do efeito trabalhador adicional de esposas referem-se às seguintes situações:

- os casais, ou membros da família ou domicílio, que vivem em um mesmo local estão igualmente expostos aos choques no mercado de trabalho que causam o desemprego do chefe do domicílio. Dessa forma, a oferta do trabalho da esposa ou de outros membros da família é menos provável, seja por restrição da própria oferta, seja por desalento devido à baixa receptividade do mercado de trabalho. Essa explicação é válida também nos casos de filhos. Entretanto, deve-se ater ao fato de que, mesmo estando todos os membros das famílias igualmente expostos aos choques, os efeitos são individuais. Em outras palavras, dependendo do conjunto de características individuais, as pessoas terão respostas diferenciadas do mercado de trabalho;

- os casais podem ter uma união do tipo assortative mating, que pressupõe que o casamento ordena os indivíduos de acordo com as características relevantes para a oferta de trabalho, tais como nível educacional e preferência por trabalho no mercado. Neste caso os casais, possivelmente, tenderão a reproduzir características semelhantes, desde que respeitadas as etapas inerentes ao ciclo de vida;

- o tempo de lazer de maridos e esposas pode ser mais complementar do que substituto. Isso é particularmente relevante para casais mais velhos, se eles consideram o desemprego do marido uma aposentadoria precoce, embora não planejada. No caso dos filhos, seu tempo de lazer só seria complementar ao do pai quando mais novos. Acima de determinada idade, possivelmente a que marca a passagem da infância para a adolescência, o tempo de lazer dos filhos tenderia a ser substituto ao dos pais;

- as mulheres podem relutar em assumir o papel de arrimo de família devido a preconceitos culturais que podem causar instabilidade emocional do chefe de família, por causa de estereótipos - como mantido pela esposa - associados a esse tipo de situação em algumas culturas. Essa explicação está muito relacionada a fatores culturais e também defasada no tempo, pois, em países desenvolvidos, onde as relações matrimoniais pressupõem um contrato de igualdade de gênero, a decisão de oferta de trabalho entre os casais está mais relacionada à produtividade de cada um no mercado ou no domicílio. Além disso, as mulheres estão cada vez mais engajadas no mercado de trabalho de forma permanente, compatibilizando, inclusive, com as funções reprodutivas. No caso dos filhos, essa explicação também não se aplica, pois existe uma cultura de apoio dos filhos em relação aos pais, que perpassa várias sociedades. Assim, pode não ser natural, em algumas sociedades, 0 chefe do sexo masculino ser mantido pela esposa. Entretanto, é normal que, independentemente do sexo, ambos sejam mantidos pelos filhos;

- a mulher pode tomar suas decisões de acordo mais com considerações dinâmicas do que estáticas, ou seja, ela pode entender que não vale a pena investir numa busca por trabalho se ela acredita que o desemprego de seu marido não durará um período tão longo, dado 
que ela sairá da ocupação assim que o marido retorne ao mercado e as condições de renda e consumo voltem ao normal no domicílio;

- em um contexto dinâmico, pode haver uma demora em conseguir casar a oferta desejada de trabalho com a demanda no mercado. Assim, o custo de oportunidade da procura torna-se alto, particularmente, se for necessário contar com arranjos alternativos de suporte e cuidados a crianças;

- programa de compensação de renda substitui ou suaviza o efeito da perda de renda e consumo, o que funciona como um fator desestimulador da entrada no mercado de trabalho. Tal explicação se aplica com grande propriedade no caso de filhos, especialmente aqueles em idade escolar. Programas de compensação de renda, de transferências de renda ou de suavização da queda da renda familiar per capita, como seguro-desemprego e Bolsa Família, podem agir contra o efeito trabalhador adicional para filhos;

- sistemas de seguridade social que providenciam renda em caso de desemprego, como são os means tested, podem desencorajar o trabalho das esposas.

Verificou-se uma escassez no Brasil de trabalhos que procuram testar a hipótese do trabalhador adicional. Schmitt e Ribeiro (2003) testam a hipótese do trabalhador adicional para as esposas da Região Metropolitana de Porto Alegre - RMPA. Os autores utilizam um modelo econométrico que isola o efeito de outras variáveis na participação das mulheres na força de trabalho. Observou-se que a hipótese do efeito trabalhador adicional é válida para explicar o comportamento das mulheres casadas no que se refere à decisão de participar da força de trabalho. No entanto, a motivação mais forte para este comportamento não é a perda do emprego do marido, mas sim a queda da renda dele.

O trabalho desenvolvido por Fernandes e Felício (2002) utiliza os painéis rotativos da PME para identificar o efeito trabalhador adicional para esposas nas regiões metropolitanas de São Paulo, Porto Alegre, Rio de Janeiro, Recife, Salvador e Belo Horizonte. Os resultados evidenciam um efeito trabalhador adicional positivo mesmo sem considerar o motivo da transição para o desemprego. Entretanto, o efeito é ainda mais significativo quando a transição do marido para o desemprego é involuntária, ou seja, por demissão de empregos formais e informais. Os autores registram ainda que o efeito estimado no trabalho é superior ao verificado para a economia americana, o que evidencia uma certa dificuldade das famílias brasileiras em adotar medidas para minimizar os efeitos das flutuações de renda e consumo em período de desemprego do chefe de família.

O estudo de Oliveira (2005), adaptando a metodologia aplicada por Fernandes e Felício (2002), estimou o efeito trabalhador para o filho mais velho com idade entre 10 e 18 anos e não identificou o efeito trabalhador adicional significativo para filhos no Brasil.

A questão da oferta de trabalho da família no Brasil metropolitano foi pioneiramente estudada por Jatobá (1990). Contudo, seu trabalho investiga a participação da família no mercado de trabalho por meio da taxa de participação da família na força de trabalho - TPFFT. Essa taxa é a razão dos membros das famílias com mais de dez anos de idade que estejam ativos sobre o total dos membros em idade ativa. O autor analisa a resposta dessa taxa ao comportamento do mercado de trabalho. Considerando que o estudo utiliza dados agregados e sem a perspectiva longitudinal, não é de natureza comparável com o presente trabalho. Ressalta-se, porém, que 
o autor identifica uma associação negativa entre a renda do chefe e a taxa de participação da família no mercado de trabalho. Isso indica que a família compensa o baixo potencial de ganho do chefe no mercado com o aumento da oferta familiar de trabalho.

Não foi encontrado nenhum trabalho na literatura nacional que investigasse a entrada de filhos no mercado de trabalho em substituição ao trabalho do chefe de família em situação de desemprego. Todavia, registra-se um estudo desenvolvido por Duryea, Lam e Levison (2003), que utiliza dados longitudinais para analisar os impactos dos choques econômicos sobre as transições da escola para o trabalho de crianças e adolescentes de 10 a 16 anos, nas seis regiões metropolitanas do Brasil cobertas pela PME. Por meio da construção de painéis rotativos com os dados da PME, similar aos painéis construídos neste trabalho, os autores compararam os domicílios nos quais os chefes homens tornaram-se desempregados durante quatro meses com os domicílios nos quais permaneceram empregados no mesmo período. As regressões de probito bivariado utilizadas no trabalho indicaram que o choque provocado pelo desemprego do chefe aumenta significativamente a probabilidade de a criança entrar no mercado de trabalho e diminui a probabilidade de a criança permanecer na escola. Os efeitos encontrados foram significativos, chegando a aumentar em 60\% a probabilidade de adolescentes de 16 anos, do sexo feminino, entrar na força de trabalho. Entre os achados, os autores destacam que os choques em anos consecutivos não apresentaram efeitos significativos, sugerindo que os resultados estão isentos de características de domicílios que experimentaram o choque de desemprego, não observáveis. Os resultados sugerem que alguns domicílios não estão aptos a absorver choques econômicos de curto prazo, o que provoca consequências negativas para as crianças.

\section{Dados e metodologia}

\section{Metodologia}

O modelo aplicado por Fernandes e Felício (2002) e adaptado por Oliveira (2005) será modificado para incorporar alterações propostas pelos autores. A pergunta para a qual se busca uma resposta é: qual seria a proporção de filhos inativos, cujos chefes de família tornaram-se desempregados, que transitariam para a força de trabalho mesmo que o chefe mantivesse a situação de empregado? A diferença entre essa taxa e a taxa real observada (chefe tornou-se desempregado) seria a medida do efeito trabalhador adicional.

Onde:

$$
\delta_{t}=\operatorname{Pr}\left(A_{i}=1 \mid D_{i}=1, L_{i}=1, t=t\right)-\operatorname{Pr}\left(A_{i}=1 \mid D_{i}=0, L_{i}=1, t=t\right)
$$

$\delta=$ efeito trabalhador adicional para filhos inativos e chefes empregados no tempo $t$, cujos chefes de família tornaram-se desempregados em $t+1$;

$A_{i}=$ variável que é igual a 1 , quando o filho da família $i$ transita para a força de trabalho no tempo $t+1$, $t+2$ ou $t+3$, e 0 quando não transita;

$D_{i}=$ variável que é igual a 1, quando o chefe da família $i$ transita para o desemprego em $t+1$, e 0 quando não transita; 
$L_{i}=$ variável que é igual a 1 para os filhos cujos chefes de família tornaram-se, de fato, desempregados em $t+1$ e 0 para aqueles filhos cujos pais permaneceram empregados em $t+1, t+2$ e $t+3$

$t=$ período de tempo no qual os filhos são observados inativos e os pais trabalhando.

Considerando $T$ o período sob análise, o efeito a ser identificado é $\delta_{T}=E\left(\delta_{t} / t \in T\right)$. Não é possível estimar concomitantemente a probabilidade de transição para a atividade de filhos cujos pais se tornaram desempregados e a mesma probabilidade na hipótese de o pai permanecer empregado. A estratégia de estimação utilizada em Fernandes e Felício (2002) e replicada em Oliveira (2005) será a mesma empregada neste trabalho, ou seja, utilizar a transição para a atividade dos filhos cujos pais não deixaram o trabalho como grupo de controle, para representar os filhos no grupo de tratamento que transitariam para a atividade mesmo se os pais não se tornassem desempregados. A validade dessa estratégia depende de quão bem esses grupos são homogêneos em uma situação não observável, na qual o chefe permanece empregado. Na verdade, pressupõe-se homogeneidade nas características individuais e familiares dos grupos refletidas no conjunto de variáveis que determinam o processo de transição dos filhos para a atividade. Se $W$ é o conjunto de variáveis relevantes, observáveis ou não, para determinar a $P_{r}\left(A_{i}=1 \mid D_{i}, t\right)$, a fórmula anterior pode ser reescrita como se segue:

$$
\begin{aligned}
& \operatorname{Pr}\left(A_{i}=1 \mid W, D_{i}=0, L_{i}=1, t=t\right)=\operatorname{Pr}\left(A_{i}=1 \mid W_{i}, D_{i}=0, L_{i}=0, t=t\right) \mathrm{e} \\
& \delta_{w t}=\operatorname{Pr}\left(A_{i}=1 \mid W, D_{i}=1, L_{i}=1, t=t\right)-\operatorname{Pr}\left(A_{i}=1 \mid W_{i}, D_{i}=0, L_{i}=0, t=t\right) \mathrm{e}
\end{aligned}
$$

Onde:

$\delta_{w t}$ é o efeito trabalhador de filhos no grupo de tratamento em $t$ com características $W$.

$$
\delta_{t}=E\left(\delta_{w t} \mid t=t\right) \text { e } \delta_{T}=E\left(\delta_{w t} \mid t \in T\right)
$$

Esta estratégia de identificação admite dois pressupostos: o primeiro é que o grupo de variáveis relevantes $W$ pode ser bem representado pelo conjunto de variáveis familiares observáveis $X$, e da região do domicílio, $r$; o segundo é que as probabilidades de transição podem ser representadas pela seguinte função que enfatiza a dependência funcional entre as probabilidades e a variável preditora $Z$ :

$$
\begin{aligned}
& \operatorname{Pr}\left(A_{i}=1\right)=\frac{1}{1+e^{-z i}} \\
& Z_{i}=\alpha+\beta D_{i}+X_{i} \Omega+\lambda_{t}+\eta_{r}
\end{aligned}
$$

Substituindo-se tem:

$$
\operatorname{Pr}\left(A_{i}=1\right)=\frac{1}{1+e^{-\left(\alpha+\beta D_{i}+X_{i} \Omega+\lambda_{i}+\eta_{i}\right)}}
$$

$X_{i}$ representa um vetor de famílias com características observáveis.

$\lambda_{t}$ e $\eta_{r}$ são variáveis controle de tempo e região.

Se o efeito trabalhador adicional existe, espera-se um sinal positivo para o coeficiente $\beta$, indicando que o fato de o chefe transitar para o desemprego aumenta a chance de o filho 
transitar para a atividade em $(\exp (\beta)-1)^{*} 100$ em relação à chance de ele não transitar. Uma vez conhecidos os coeficientes estimados do modelo, pode-se obter, para cada amostra de filhos, a estimativa da probabilidade de transição para o mercado de trabalho nas situações nas quais $D_{i}=j(j=0,1)$, mantidas constantes as características da amostra. Assim, $\hat{\delta}_{T}=\hat{P}_{1}-\hat{P}_{0}$, $\hat{P}_{j}$ são as probabilidades médias de transição estimadas para filhos cujos pais tiveram sua experiência de transição em $D_{i}=j$.

A propriedade da função logística definida anteriormente é que, quando $Z$ se torna infinitesalmente negativo, $e^{-z}$ se torna infinitesalmente grande e, assim, a probabilidade de transição aproxima-se de zero. Quando $Z$ se torna infinitesalmente positivo, $e^{-Z}$ se torna infinitesalmente pequeno, logo, a probabilidade de transição para a atividade aproxima-se da unidade. Se $Z=0, e^{-Z}=1$ e a probabilidade de transição é igual a 0,5.

\section{Variáveis selecionadas e os modelos testados}

Boa parte dos resultados obtidos por meio de qualquer modelo implementado está na definição do modelo, ou seja, quais serão as variáveis explicativas que produzem efeitos significantes sobre a variável resposta. Nesse caso, além das tradicionais variáveis usadas nos modelos de participação na força de trabalho, selecionaram-se aquelas que foram julgadas mais relevantes sobre a transição de crianças e adolescentes para a atividade.

QUADRO 1

Variáveis selecionadas para as regressões

\begin{tabular}{|c|c|c|c|c|c|}
\hline Código & Descrição & Categorias & Mod. 1 & Mod. 2 & Mod. 3 \\
\hline D & $\begin{array}{l}\text { Transição do chefe para } \\
\text { o desemprego }\end{array}$ & $\begin{array}{l}\text { 1: Se o chefe estava ativo em T zero e } \\
\text { transitou para o desemprego } T+1 \text {. } \\
0: \text { Caso contrário }\end{array}$ & $x$ & $x$ & $x$ \\
\hline A & $\begin{array}{l}\text { Transição do filho para a } \\
\text { atividade }\end{array}$ & $\begin{array}{l}\text { 1: Se o filho estava inativo em T zero e } \\
\text { transitou para a atividade em } T+1, T+2 \\
\text { ou } T+3 \text {. } \\
0: \text { Caso contrário }\end{array}$ & & $x$ & $x$ \\
\hline PaiDesemp & Chefe desempregado & $\begin{array}{l}\text { 1: Se o chefe estava desempregado } \\
\text { em algum ponto das quatro primeiras } \\
\text { entrevistas } \\
\text { 0: Caso contrário }\end{array}$ & & & $x$ \\
\hline FilhoAtivo & Filho ativo em T zero & $\begin{array}{l}\text { 1: Se o filho esteve ativo em algum ponto } \\
\text { das quatro primeiras entrevistas } \\
\text { 0: Caso contrário }\end{array}$ & & & $x$ \\
\hline SP & Dummy São Paulo & $\begin{array}{l}\text { 1: Região Metropolitana de São Paulo } \\
\text { 0: Caso contrário }\end{array}$ & & & \\
\hline PE & Dummy Recife & $\begin{array}{l}\text { 1: Região Metropolitana de Recife } \\
\text { 0: Caso contrário }\end{array}$ & & $x$ & $x$ \\
\hline BA & Dummy Salvador & $\begin{array}{l}\text { 1: Região Metropolitana de Salvador } \\
\text { 0: Caso contrário }\end{array}$ & & $x$ & $x$ \\
\hline MG & Dummy Belo Horizonte & $\begin{array}{l}\text { 1: Região Metropolitana de Belo } \\
\text { Horizonte } \\
\text { 0: Caso contrário }\end{array}$ & & $x$ & $x$ \\
\hline RJ & Dummy Rio de Janeiro & $\begin{array}{l}\text { 1: Região Metropolitana do Rio de Janeiro } \\
\text { 0: Caso contrário }\end{array}$ & & $x$ & $x$ \\
\hline RS & Dummy Porto Alegre & $\begin{array}{l}\text { 1: Região Metropolitana de Porto Alegre } \\
\text { 0: Caso contrário }\end{array}$ & & $x$ & $x$ \\
\hline
\end{tabular}


...continuação

\begin{tabular}{|c|c|c|c|c|c|}
\hline Código & Descrição & Categorias & Mod. 1 & Mod. 2 & Mod. 3 \\
\hline A2002 & Dummy 2002 & $\begin{array}{l}\text { 1: Pesquisa realizada em } 2002 \\
\text { 0: Caso contrário }\end{array}$ & & & \\
\hline A2003 & Dummy 2003 & $\begin{array}{l}\text { 1: Pesquisa realizada em } 2003 \\
\text { 0: Caso contrário }\end{array}$ & & $x$ & $x$ \\
\hline A2004 & Dummy 2004 & $\begin{array}{l}\text { 1: Pesquisa realizada em } 2004 \\
\text { 0: Caso contrário }\end{array}$ & & $x$ & $x$ \\
\hline A2005 & Dummy 2005 & $\begin{array}{l}\text { 1: Pesquisa realizada em } 2005 \\
\text { 0: Caso contrário }\end{array}$ & & $x$ & $x$ \\
\hline A2006 & Dummy 2006 & $\begin{array}{l}\text { 1: Pesquisa realizada em } 2006 \\
\text { 0: Caso contrário }\end{array}$ & & $x$ & $x$ \\
\hline A2007 & Dummy 2007 & $\begin{array}{l}\text { 1: Pesquisa realizada em } 2007 \\
\text { 0: Caso contrário }\end{array}$ & & $x$ & $x$ \\
\hline A2008 & Dummy 2008 & $\begin{array}{l}\text { 1: Pesquisa realizada em } 2008 \\
\text { 0: Caso contrário }\end{array}$ & & $x$ & $x$ \\
\hline A2009 & Dummy 2009 & $\begin{array}{l}\text { 1: Pesquisa realizada em } 2009 \\
\text { 0: Caso contrário }\end{array}$ & & $x$ & $x$ \\
\hline A2010 & Dummy 2010 & $\begin{array}{l}\text { 1: Pesquisa realizada em } 2010 \\
\text { 0: Caso contrário }\end{array}$ & & $x$ & $x$ \\
\hline A2011 & Dummy 2011 & $\begin{array}{l}\text { 1: Pesquisa realizada em } 2011 \\
\text { 0: Caso contrário }\end{array}$ & & $x$ & $x$ \\
\hline A2012 & Dummy 2012 & $\begin{array}{l}\text { 1: Pesquisa realizada em } 2012 \\
\text { 0: Caso contrário }\end{array}$ & & $x$ & $x$ \\
\hline A2013 & Dummy 2013 & $\begin{array}{l}\text { 1: Pesquisa realizada em } 2013 \\
\text { 0: Caso contrário }\end{array}$ & & $x$ & $x$ \\
\hline Informal & $\begin{array}{l}\text { Ocupação do chefe } \\
\text { segundo a informalidade }\end{array}$ & $\begin{array}{l}\text { 1: Chefe empregado sem carteira ou } \\
\text { conta-própria } \\
\text { 0: Caso contrário }\end{array}$ & & $x$ & $x$ \\
\hline TotalPes & $\begin{array}{l}\text { Total de pessoas na } \\
\text { família }\end{array}$ & Variável contínua & & $x$ & $x$ \\
\hline AnestPai & $\begin{array}{l}\text { Anos de estudo do pai/ } \\
\text { mãe }\end{array}$ & Variável contínua & & $x$ & $x$ \\
\hline ProPad & $\begin{array}{l}\text { Proporção de filhos } \\
\text { maiores de } 18 \text { anos no } \\
\text { domicílio }\end{array}$ & Variável contínua (0 a 1) & & $x$ & $x$ \\
\hline SexFil & Sexo do filho & $\begin{array}{l}\text { 1: Homem } \\
\text { 2: Mulher }\end{array}$ & & $x$ & $x$ \\
\hline IdadeFil & Idade do filho mais velho & Variável contínua & & $x$ & $x$ \\
\hline idadeFil2 & $\begin{array}{l}\text { Idade do filho mais velho } \\
\text { ao quadrado }\end{array}$ & Variável contínua & & $x$ & $x$ \\
\hline
\end{tabular}

Como os modelos estão aninhados, ou seja, o terceiro incorpora o segundo que incorpora o primeiro, pode-se testar se a adição de variáveis de controle melhora o ajuste do modelo.

Os modelos podem ser especificados da seguinte forma:

Modelo genérico $\operatorname{Pr}\left(A_{i}=1\right)=\frac{1}{1+e^{-\left(\alpha+\beta D+\Omega_{i} X+\lambda_{j} \mathrm{Y}+\eta_{k} \mathrm{Z}\right)}}$

Considerando que $X$ representa um vetor de famílias com características observáveis e $Y$ e $Z$ representam os vetores de tempo e região, a estatística para a qual se conhece a distribuição da amostra é:

$$
\begin{aligned}
-\log \left(\mathrm{L}_{1} / \mathrm{L}_{2}\right)^{2} & =-2 \log \left(\mathrm{L}_{1} / \mathrm{L}_{2}\right) \\
& =-2\left(\log \mathrm{L}_{1} / \log \mathrm{L}_{2}\right) \\
& =\left(-2 \log \mathrm{L}_{1}\right)-\left(-2 \log \mathrm{L}_{2}\right)
\end{aligned}
$$


$L_{1} / L_{2}$ é a razão de verossimilhança e $L_{1}<L_{2}$ não é uma condição restritiva, apenas indica que o modelo 1 está aninhado no modelo 2. O teste é um qui-quadrado, assim, tomando-se os graus de liberdade como a diferença entre o número de coeficientes estimados, consulta-se a tabela de qui-quadrado para verificar se um ajuste é significativamente melhor do que o outro.

Um indicador do ajuste do modelo que pode ser calculado é o pseudo-R² utilizando as estatísticas geradas na saída do software estatístico.

pseudo $-R^{2}=\frac{2 \log L_{1}-2 \log L_{0}-2 k}{-2 \log L_{0}}$

$L_{0}$ é a verossimilhança para o ajuste do modelo nulo.

$L_{1}$ é a verossimilhança para o modelo cujo ajuste está sendo testado.

\section{Dados}

A PME é um levantamento estatístico sobre o mercado de trabalho metropolitano no Brasil, cujo objetivo fundamental é fornecer indicadores mensais de emprego e desemprego. A pesquisa revela tendências dos mercados de trabalho de algumas regiões metropolitanas (RM) - Rio de Janeiro, São Paulo, Belo Horizonte, Porto Alegre, Recife e Salvador -, com base em informações conjunturais. Apesar de a cobertura não ser nacional, considera-se que o comportamento desses mercados de trabalho metropolitano sinalize as condições mais gerais de funcionamento da economia. Em 2002, foram realizadas algumas alterações na pesquisa seguindo recomendações internacionais em termos de conceitos sobre o tema trabalho; ampliação da investigação e por conseguinte do conhecimento sobre a População Economicamente Ativa (PEA) e da oferta potencial de trabalho; ajustamento no processo de rotação para aprimorar o acompanhamento longitudinal de eventos; e introdução do coletor eletrônico para aprimorar o sistema operacional e agilizar a apuração dos resultados da pesquisa (IBGE, 2002).

Para o cálculo de seus indicadores são utilizadas informações levantadas por meio de uma amostra probabilística de domicílios estratificada e conglomerada em dois estágios para cada RM de abrangência da pesquisa. Os setores censitários constituem as unidades primárias de amostragem (UPA) e são selecionados por meio de amostragem sistemática proporcional ao total de domicílios particulares ocupados verificados no Censo Demográfico do início da década. O setor selecionado permanece na amostra até o próximo Censo, quando se refaz todo o processo de seleção. As unidades secundárias são os domicílios de cada setor censitário. Os domicílios são selecionados por meio de amostragem sistemática simples, com base em um cadastro de domicílios de cada setor que é mantido atualizado. 0 número de unidades domiciliares selecionadas para integrar a amostra total é de aproximadamente 40.000 a cada mês. As unidades domiciliares amostradas são distribuídas pelas quatro semanas de referência da pesquisa e o resultado do mês é obtido pela média dessas quatro semanas.

Trata-se, portanto, de um processo de amostragem complexa e as estimativas geradas a partir destes dados devem considerar o desenho amostral. Para minimizar o efeito do plano amostral nas estimativas, foi gerado um plano para análise de dados de amostras complexas 
no SPSS. A variável de estratificação (v112), a UPA (v113) e o peso da pessoa com correção de não entrevista com calibração pela projeção de população foram usados no primeiro estágio, seguidos pela probabilidade do setor (v107) e o tamanho da população (v114). O método de correção foi amostra probabilística igual sem reposição (WOR). Pessoa e Silva (1998, p. 10) discutem os efeitos sobre as estimativas geradas quando se ignora o desenho amostral:

As estimativas pontuais de parâmetros da população são influenciadas por pesos distintos das observações. Além disso, as estimativas de variância são influenciadas pela conglomeração, estratificação e pesos. Ao ignorar estes aspectos, os pacotes tradicionais de análise podem produzir estimativas incorretas das variâncias das estimativas pontuais.

A PME permite a construção de painéis, pois cada unidade domiciliar selecionada é pesquisada durante quatro meses consecutivos, fica oito meses sem ser pesquisada e, após esse período, volta por mais quatro meses ao painel, quando é definitivamente retirada. A amostra é subdividida em oito painéis de rotação, em que, a cada mês, 25\% dos domicílios da amostra são substituídos, seguindo um esquema de grupos de rotação, compostos por um conjunto de setores censitários, e painéis formados por um conjunto e unidades domiciliares (IBGE, 2002). Em consequência desse esquema de rotação, para o mesmo mês, em pares de anos consecutivos, são garantidos 50\% de parte comum da amostra (IBGE, 2002).

A amostra do presente estudo é formada por filhos na idade de 10 a 18 anos inativos na quinta entrevista e por chefes ocupados na quinta entrevista entre 2002 e 2013 . 0 grupo de tratamento é composto pelos filhos que transitaram para a atividade entre a sexta e a oitava entrevistas e cujos chefes transitaram da situação de ocupados para desempregados entre a quinta e a sexta entrevistas. 0 grupo de controle é formado pelos filhos que transitaram para a atividade entre a sexta e a oitava entrevistas, cujos chefes permaneceram empregados nesse mesmo intervalo de tempo. Outros casos foram excluídos da amostra.

O banco de dados construído, segundo as restrições anteriores, resultou numa amostra de 52.299.500 pares de chefes e filhos. Entre os chefes, 36.078.384 são homens, dos quais $275.967(0,8 \%)$ transitaram para o desemprego entre a quinta e a sexta entrevistas, e 16.221.116 são mulheres, com o mesmo valor relativo de transição para o desemprego (0,8\%), mas, em termos absolutos, representando apenas 121.674 .

Da amostra total de filhos, 4.529 .759 (9,0\%) transitaram para a atividade entre $t+1$ e $t+4$ e o restante (47.769.742, ou 91,0\%) não transitou.

0 grupo de controle, que representa o total de filhos que transitaram para a atividade em $t+1, t+2$ ou $t+3$ cujos chefes de família mantiveram-se empregados em todo o período, englobou 4.471.219 indivíduos. O grupo de tratamento - filhos que transitaram para a atividade entre $t+1$ e $t+3$, cujos pais efetivamente tornaram-se desempregados em $t+1$ - é formado por 58.539 indivíduos. Quando os dados são desagregados por sexo do chefe, observa-se que apenas 35\% dos indivíduos pertencentes ao grupo de controle são membros de família chefiada por mulher. Assim, os dados sinalizam que os filhos transitam para a atividade, em grande maioria, independentemente da transição do chefe de família para o desemprego. 


\section{Resultados}

Foram estimados três modelos para os chefes do sexo masculino e três modelos para os chefes do sexo feminino. A Tabela 1 apresenta os coeficientes estimados para o efeito da transição dos chefes homens sobre a transição dos filhos de 10 a 18 anos para a atividade. No primeiro modelo, não foram incluídas variáveis de controle, apenas aquela que indica se o chefe transitou para o desemprego no tempo $t+1$. No segundo modelo, foram incluídas as variáveis de características demográficas e individuais, bem como variáveis dummies de UFe dos períodos de realização da pesquisa. No terceiro modelo foram acrescidas duas varáveis que refletem a situação ocupacional do chefe e do filho: se o chefe esteve desempregado, considerando os quatro primeiros pontos de entrevista que antecedem o período de transição; e se o filho esteve ativo no mesmo período.

Nos três modelos estimados, a variável de transição do chefe para o desemprego foi positiva e significante estatisticamente, ou seja, o fato de o chefe do sexo masculino transitar para o desemprego aumenta a chance de o filho se tornar ativo. $O$ fato de o filho ser homem tem chance $11 \%$ maior de se tornar ativo diante do desemprego do pai em relação às filhas. Este resultado é coerente com o perfil de entrada de homens e mulheres no mercado de trabalho.

Se o pai esteve em uma relação informal de trabalho em um dos primeiros quatro pontos da entrevista, isso também tem o efeito de aumentar a probabilidade de o filho transitar para o mercado de trabalho. Os anos de realização da pesquisa só não foram significantes, tanto no modelo 2 quanto no 3, em 2003, 2005 e 2007, com todos os coeficientes negativos indicando efeito de diminuição da probabilidade de o filho ingressar no mercado de trabalho, em relação ao ano de 2002, omitido na regressão. Neste período verificou-se grande expansão do emprego formal e da renda no Brasil, o que pode indicar baixa transição involuntária do chefe para o desemprego e, em consequência, menor necessidade de ingresso dos filhos no mercado de trabalho para complementar a renda familiar.

A RM de Porto Alegre foi a única que não apresentou significância para o efeito investigado. As demais foram significativas, com coeficientes negativos nas RMs de Salvador e do Rio de Janeiro e positivos nas de Belo Horizonte e de Recife. Assim, é mais provável que o filho transite para o mercado de trabalho para compensar a perda de emprego do pai nas RMs de Recife e de Belo Horizonte, em comparação com a RM de São Paulo (referência).

As variáveis totalpes (total de pessoas na família) e propad (proporção de filhos com idade superior a 18 anos) são significantes e negativas, ou seja, impactam diminuindo a probabilidade de transição do filho para o mercado de trabalho, nos modelos 2 e 3 . Este é um resultado esperado, pois, quanto mais pessoas na família, mais oferta de trabalho dos membros mais velhos, inclusive com idade superior a 18 anos, que poderiam ingressar ou já estariam economicamente ativos e arrefeceriam a necessidade da entrada de membros mais novos no mercado de trabalho, para suprir a queda da renda familiar no caso do desemprego do chefe. 
TABELA 1

Resultados das regressões logísticas para estimar o efeito trabalhador adicional para filhos de chefes de família do sexo masculino

Regiões metropolitanas de Belo Horizonte, Salvador, Porto Alegre, São Paulo, Rio de Janeiro e Recife - 2002-2013

\begin{tabular}{|c|c|c|c|c|c|c|c|c|c|c|c|c|}
\hline \multirow[b]{2}{*}{ Variáveis } & \multicolumn{4}{|c|}{ Modelo 1} & \multicolumn{4}{|c|}{ Modelo 2} & \multicolumn{4}{|c|}{ Modelo 3} \\
\hline & Coef. & valor & $\begin{array}{l}\text { Exp. } \\
\text { (coef.) }\end{array}$ & EPA & Coef. & $\begin{array}{l}P_{-} \\
\text {valor }\end{array}$ & $\begin{array}{l}\text { Exp. } \\
\text { (coef.) }\end{array}$ & EPA & Coef. & valor & $\begin{array}{l}\text { Exp. } \\
\text { (coef.) }\end{array}$ & EPA \\
\hline Constante & $-2,432$ & 0,000 & 0,088 & 1,981 & $-7,229$ & 0,000 & 0,001 & 1,440 & $-6,828$ & 0,000 & 0,001 & 1,429 \\
\hline $\begin{array}{l}\text { Transiçãa do } \\
\text { chefe para o } \\
\text { desemprego }\end{array}$ & 0,768 & 0,000 & 2,157 & 1,616 & 0,637 & 0,000 & 1,891 & 1,398 & 0,559 & 0,000 & 1,748 & 1,321 \\
\hline Sexo do filho & & & & & 0,104 & 0,003 & 1,110 & 1,225 & 0,075 & 0,032 & 1,078 & 1,237 \\
\hline $\begin{array}{l}\text { Ocupação do } \\
\text { chefe segundo }\end{array}$ & & & & & & & & & & & & \\
\hline a informalidade & & & & & 0,110 & 0,003 & 1,117 & 1,360 & 0,078 & 0,045 & 1,081 & 1,384 \\
\hline Dummy 2003 & & & & & 0,015 & 0,847 & 1,015 & 1,361 & $-0,019$ & 0,805 & 0,981 & 1,367 \\
\hline Dummy 2004 & & & & & $-0,256$ & 0,002 & 0,774 & 1,400 & $-0,276$ & 0,001 & 0,758 & 1,394 \\
\hline Dummy 2005 & & & & & $-0,042$ & 0,611 & 0,959 & 1,444 & $-0,041$ & 0,616 & 0,960 & 1,449 \\
\hline Dummy 2006 & & & & & $-0,165$ & 0,042 & 0,848 & 1,378 & $-0,166$ & 0,043 & 0,847 & 1,381 \\
\hline Dummy 2007 & & & & & $-0,137$ & 0,099 & 0,872 & 1,443 & $-0,135$ & 0,107 & 0,874 & 1,446 \\
\hline Dummy 2008 & & & & & $-0,314$ & 0,000 & 0,731 & 1,433 & $-0,302$ & 0,001 & 0,739 & 1,440 \\
\hline Dummy 2009 & & & & & $-0,202$ & 0,023 & 0,817 & 1,453 & $-0,183$ & 0,041 & 0,833 & 1,454 \\
\hline Dummy 2010 & & & & & $-0,288$ & 0,002 & 0,750 & 1,437 & $-0,276$ & 0,003 & 0,759 & 1,459 \\
\hline Dummy 2011 & & & & & $-0,267$ & 0,003 & 0,766 & 1,408 & $-0,247$ & 0,007 & 0,781 & 1,411 \\
\hline Dummy 2012 & & & & & $-0,368$ & 0,002 & 0,692 & 1,492 & $-0,343$ & 0,004 & 0,710 & 1,494 \\
\hline $\begin{array}{l}\text { Dummy RM } \\
\text { Recife }\end{array}$ & & & & & 0,369 & 0,000 & 1,447 & 0,810 & 0,418 & 0,000 & 1,518 & 0,807 \\
\hline $\begin{array}{l}\text { Dummy RM } \\
\text { Salvador }\end{array}$ & & & & & -0.263 & 0,000 & 0,769 & 0,865 & $-0,191$ & 0008 & 0826 & 0851 \\
\hline Dummy RM & & & & & $-0,203$ & 0,000 & $0, / 69$ & 0,805 & $-0,191$ & 0,008 & $0,8<0$ & 0,851 \\
\hline Belo Horizonte & & & & & 0,306 & 0,000 & 1,358 & 0,982 & 0,308 & 0,000 & 1,361 & 0,960 \\
\hline $\begin{array}{l}\text { Dummy RM } \\
\text { Rio de Janeiro }\end{array}$ & & & & & $-0,964$ & 0,000 & 0,381 & 2,092 & $-0,848$ & 0,000 & 0,428 & 2,006 \\
\hline $\begin{array}{l}\text { Dummy RM } \\
\text { Porto Alegre }\end{array}$ & & & & & 0,043 & 0,435 & 1,044 & 0,921 & 0,061 & 0.273 & 1.062 & 0,902 \\
\hline $\begin{array}{l}\text { Anos de estudo } \\
\text { do pai/mãe }\end{array}$ & & & & & $-0,030$ & 0,000 & 0,971 & 1,415 & $-0,022$ & 0,000 & 0,979 & 1,369 \\
\hline $\begin{array}{l}\text { Total de pessoas } \\
\text { na família }\end{array}$ & & & & & $-0,040$ & 0,008 & 0,961 & 1,329 & $-0,029$ & 0,056 & 0,972 & 1,333 \\
\hline $\begin{array}{l}\text { Proporção de } \\
\text { filhos maiores d }\end{array}$ & & & & & & & & & & & & \\
\hline $\begin{array}{l}18 \text { anos no dom } \\
\text { Idade do filho } \mathrm{m}\end{array}$ & $\begin{array}{l}\text { nicílio } \\
\text { hais }\end{array}$ & & & & $-0,397$ & 0,000 & 0,672 & 1,352 & $-0,556$ & 0,000 & 0,574 & 1,359 \\
\hline velho & & & & & 0,500 & 0,000 & 1,648 & 1,456 & 0,434 & 0,000 & 1,543 & 1,434 \\
\hline $\begin{array}{l}\text { Idade do filho } \mathrm{m} \\
\text { velho ao quadra }\end{array}$ & & & & & $-0,010$ & 0,000 & 0,990 & 1,450 & $-0,008$ & 0,000 & 0,992 & 1,431 \\
\hline $\begin{array}{l}\text { Chefe } \\
\text { desempregado }\end{array}$ & & & & & & & & & 0,161 & 0,048 & 1,174 & 1,268 \\
\hline $\begin{array}{l}\text { Filho ativo em } \\
\text { Tzero }\end{array}$ & & & & & & & & & 0,608 & 0,000 & 1,836 & 1,189 \\
\hline $\begin{array}{l}\text { Tamanho da } \\
\text { amostra }\end{array}$ & & & & & 36.0 & 78.384 & & & 27.2 & 38.196 & 27.2 & 38.196 \\
\hline $\begin{array}{l}\text { Teste } \\
\text { qui-quadrado }\end{array}$ & & & & & & 29,191 & & & & 56,534 & & 56,483 \\
\hline Pseudo $R^{2}$ & & & & & & 0,000 & & & & 0,000 & & 0,000 \\
\hline
\end{tabular}

Fonte: IBGE. Microdados PME de 2002 a 2013. 
A idadefil (idade do filho mais velho) é significante e tem coeficiente positivo, indicando que a cada incremento de uma unidade na idade do filho mais velho aumenta em $64,8 \%$ no modelo 2 e em 54,3\% no modelo 3 a chance de o filho de 10 a 18 anos entrar no mercado de trabalho. Na hipótese de o filho mais velho ter menos de 18 anos, é de se esperar que a probabilidade aumente com a idade. Por outro lado, se o filho mais velho tiver mais de 18 anos, o limite da oferta de trabalho em benefício da renda familiar é a transição para o casamento, formando outro núcleo familiar, o que pode indicar maior mobilização de recursos em favor dessa transição em prejuízo da mobilização na unidade familiar.

A variável anos de estudos do pai, como esperado, apresenta relação negativa com a variável resposta, ou seja, o incremento de um ano de escolaridade do pai produz efeito de diminuir em 2,9\% e 2,1\% a probabilidade de ingresso do filho de 10 a 18 anos no mercado de trabalho, respectivamente, modelos 2 e 3.

A variável paidesem do modelo 3, que representa alguma situação de desemprego do pai em um dos quatro primeiro pontos do painel, aumenta o espaço de tempo de transição do chefe para o desemprego e eleva em 17,4\% a probabilidade de transição do filho para o mercado de trabalho. Situações de atividade do filho em alguma das quatro primeiras entrevistas é significante e produz alto impacto na probabilidade de transição do filho para a atividade. Ele tem chance $83,6 \%$ maior de ingressar no mercado de trabalho do que um filho que não tenha experimentado estados de atividade anteriores. Essas duas variáveis do modelo 3 refletem o histórico de participação dos pais e filhos no mercado de trabalho e podem minimizar o viés de seleção que poderia impactar na transição, tal como preferência por lazer não observável.

A Tabela 2 apresenta os resultados dos três modelos estimados considerando apenas as chefes de família. Neste caso, a variável de transição do chefe para o desemprego não foi estatisticamente significativa em nenhum dos três modelos, indicando que essa variável, neste caso, é um fraco preditor da transição dos filhos de 10 a 18 anos para o mercado de trabalho.

A maior parte das variáveis que foram fortes preditoras no modelo dos chefes homens não foi significante em nenhum dos modelos para as mulheres chefes de família. Variáveis indicadoras de ano da pesquisa foram significantes a partir de 2006 em relação a 2002 e apresentaram coeficientes negativos, diminuindo a probabilidade de entrada dos filhos, nas idades investigadas, no mercado de trabalho. No caso das RMs de residência, os resultados foram similares aos dos chefes homens em significância e sinal dos coeficientes.

A variável anestpai (anos de estudos do chefe de família) é negativa e significante estatisticamente para chefes mulheres, assim como foi para os homens, e produz o resultado esperado, ou seja, uma maior escolaridade do chefe de família diminui a chance de transição do filho para a atividade. 
TABELA 2

Resultados das regressões logísticas para estimar o efeito trabalhador adicional para filhos de chefes de família do sexo feminino

Regiões metropolitanas de Belo Horizonte, Salvador, Porto Alegre, São Paulo, Rio de Janeiro e Recife - 2002-2013

\begin{tabular}{|c|c|c|c|c|c|c|c|c|c|c|c|c|}
\hline \multirow[b]{2}{*}{ Variáveis } & \multicolumn{4}{|c|}{ Modelo 1} & \multicolumn{4}{|c|}{ Modelo 2} & \multicolumn{4}{|c|}{ Modelo 3} \\
\hline & Coef. & ${ }_{\text {valor }}^{\mathrm{P}_{-}}$ & $\begin{array}{l}\text { Exp. } \\
\text { (coef.) }\end{array}$ & EPA & Coef. & ${ }_{\text {valor }}^{\mathrm{P}_{-}}$ & $\begin{array}{l}\text { Exp. } \\
\text { (coef.) }\end{array}$ & EPA & Coef. & ${ }_{\text {valor }}^{\mathrm{P}_{-}}$ & $\begin{array}{l}\text { Exp. } \\
\text { (coef.) }\end{array}$ & EPA \\
\hline Constante & $-2,219$ & 0,000 & 0,109 & 1,396 & $-3,035$ & 0,001 & 0,048 & 2,182 & $-3,000$ & 0,000 & 0,050 & 2,075 \\
\hline $\begin{array}{l}\text { Transição do } \\
\text { chefe para o }\end{array}$ & & & & & & & & & & & & \\
\hline desemprego & 0,224 & 0,225 & 1,251 & 0,947 & 0,224 & 0,352 & 1,251 & 0,978 & 0,222 & 0,364 & 1,248 & 0,983 \\
\hline Sexo do filho & & & & & $-0,026$ & 0,615 & 0,974 & 1,115 & $-0,051$ & 0,324 & 0,950 & 1,114 \\
\hline $\begin{array}{l}\text { Ocupação do } \\
\text { chefe segundo } \\
\text { a informalidade }\end{array}$ & & & & & $-0,036$ & 0,567 & 0,964 & 1,346 & $-0,071$ & 0,283 & 0,931 & 1,390 \\
\hline Dummy 2003 & & & & & $-0,090$ & 0,495 & 0,914 & 1,317 & $-0,136$ & 0,306 & 0,873 & 1,308 \\
\hline Dummy 2004 & & & & & $-0,238$ & 0,082 & 0,788 & 1,357 & $-0,269$ & 0,049 & 0,764 & 1,344 \\
\hline Dummy 2005 & & & & & $-0,098$ & 0,456 & 0,907 & 1,320 & $-0,105$ & 0,425 & 0,900 & 1,319 \\
\hline Dummy 2006 & & & & & $-0,256$ & 0,048 & 0,774 & 1,248 & $-0,271$ & 0,036 & 0,763 & 1,248 \\
\hline Dummy 2007 & & & & & $-0,289$ & 0,033 & 0,749 & 1,323 & $-0,303$ & 0,026 & 0,738 & 1,317 \\
\hline Dummy 2008 & & & & & $-0,360$ & 0,009 & 0,698 & 1,341 & $-0,355$ & 0,010 & 0,701 & 1,337 \\
\hline Dummy 2009 & & & & & $-0,479$ & 0,001 & 0,620 & 1,434 & $-0,467$ & 0,001 & 0,627 & 1,391 \\
\hline Dummy 2010 & & & & & $-0,368$ & 0,007 & 0,692 & 1,326 & $-0,348$ & 0,012 & 0,706 & 1,329 \\
\hline Dummy 2011 & & & & & $-0,406$ & 0,006 & 0,666 & 1,434 & $-0,378$ & 0,009 & 0,685 & 1,410 \\
\hline Dummy 2012 & & & & & $-0,381$ & 0,016 & 0,683 & 1,293 & $-0,351$ & 0,028 & 0,704 & 1,294 \\
\hline $\begin{array}{l}\text { Dummy RM } \\
\text { Recife }\end{array}$ & & & & & 0,499 & 0,000 & 1,648 & 0,919 & 0,550 & 0,000 & 1,733 & 0,933 \\
\hline $\begin{array}{l}\text { Dummy RM } \\
\text { Salvador }\end{array}$ & & & & & $-0,190$ & 0,038 & 0,827 & 0,824 & $-0,130$ & 0,155 & 0,878 & 0,819 \\
\hline $\begin{array}{l}\text { Dummy RM } \\
\text { Belo Horizonte }\end{array}$ & & & & & 0,415 & 0,000 & 1,515 & 1,039 & 0,417 & 0,000 & 1,518 & 1,028 \\
\hline $\begin{array}{l}\text { Dummy RM } \\
\text { Rio de Janeiro }\end{array}$ & & & & & $-0,795$ & 0,000 & 0,452 & 1,603 & $-0,686$ & 0,000 & 0,504 & 1,590 \\
\hline $\begin{array}{l}\text { Dummy RM } \\
\text { Porto Alegre }\end{array}$ & & & & & 0,076 & 0,345 & 1,079 & 0,836 & 0,092 & 0,255 & 1,097 & 0,839 \\
\hline $\begin{array}{l}\text { Anos de } \\
\text { estudo do } \\
\text { pai/mãe }\end{array}$ & & & & & $-0,033$ & 0,000 & 0,968 & 1,371 & $-0,026$ & 0,001 & 0,974 & 1,361 \\
\hline Total de & & & & & & & & & & & & \\
\hline $\begin{array}{l}\text { pessoas } \\
\text { na família }\end{array}$ & & & & & $-0,022$ & 0,368 & 0,978 & 1,510 & $-0,011$ & 0,624 & 0,989 & 1,427 \\
\hline $\begin{array}{l}\text { Proporção de } \\
\text { filhos maiores } \\
\text { de } 18 \text { anos no } \\
\text { domicílio }\end{array}$ & & & & & 0,044 & 0,880 & 1,045 & 1,886 & $-0,130$ & 0,459 & 0,878 & 1,521 \\
\hline $\begin{array}{l}\text { Idade do filho } \\
\text { mais velho }\end{array}$ & & & & & 0,105 & 0,456 & 1,111 & 2,211 & 0,080 & 0,238 & 1,083 & 2,171 \\
\hline $\begin{array}{l}\text { Idade do filho } \\
\text { mais velho ao } \\
\text { quadrado }\end{array}$ & & & & & $-0,001$ & 0,707 & 0,999 & 2,225 & $-0,001$ & 0,632 & 0,999 & 2,220 \\
\hline $\begin{array}{l}\text { Chefe } \\
\text { desempregado }\end{array}$ & & & & & & & & & 0,164 & 0,116 & 1,178 & 1,249 \\
\hline $\begin{array}{l}\text { Filho ativo } \\
\text { em Tzero }\end{array}$ & & & & & & & & & 0,611 & 0,000 & 1,843 & 1,255 \\
\hline $\begin{array}{l}\text { Tamanho } \\
\text { da amostra }\end{array}$ & & & 16.2 & 21.116 & & & & 155.273 & & & & 55.273 \\
\hline $\begin{array}{l}\text { Teste } \\
\text { qui-quadrado }\end{array}$ & & & & 1,472 & & & & 02,198 & & & & 29,251 \\
\hline Pseudo $\mathrm{R}^{2}$ & & & & 0,000 & & & & 0,000 & & & & 0,000 \\
\hline
\end{tabular}

Fonte: IBGE. Microdados PME de 2002 a 2013. 
Ressalta-se o efeito significante da variável de atividade dos filhos em um dos quatro primeiros pontos da entrevista incluído no modelo 3, com coeficiente positivo, aumentando em $84,3 \%$ a chance de ingresso no mercado de trabalho. Vale refletir sobre o que representam o fraco efeito da variável de transição e de outras variáveis e o forte poder de predição da variável filho ativo. Uma hipótese pode ser a que associa pobreza à chefia feminina de família e domicílios. Assim, os filhos de 10 a 18 anos membros destas famílias já transitariam para a atividade em virtude da necessidade de complementação da renda familiar. Por isso o forte poder de predição da variável que reflete o histórico de atividade do filho.

O pseudo- $R^{2}$ estimado indica fraco ajuste, revelando o baixo poder preditivo das variáveis explicativas sobre a variável resposta, embora a maior parte das variáveis incluídas nos modelos tenha sido estatisticamente significante. Contudo, sabe-se que o significado desta estatística neste tipo de modelo é bastante difuso.

O valor estimado a partir das diferenças entre as médias das probabilidades de transição para os grupos de tratamento e controle revela a existência de um efeito trabalhador adicional positivo (Tabela 3). Note-se que o efeito da transição do chefe homem para o desemprego é superior ao verificado para os chefes do sexo feminino nos três modelos. Este achado reforça a hipótese levantada sobre o fraco pode preditor da variável de transição da mulher chefe para o desemprego. Quando o homem é o principal provedor, a transição dos filhos para a atividade acontece mais em decorrência da própria transição de ciclo de vida e em função da queda na renda familiar na falta do salário do provedor. Já no caso das mulheres, a transição ocorre em função da carência material e em menor proporção pelo desemprego.

TABELA 3

Estimativas do efeito trabalhador adicional

Regiões metropolitanas de Belo Horizonte, Salvador, Porto Alegre, São Paulo, Rio de Janeiro e Recife - 2002-2013

\begin{tabular}{|c|c|c|c|c|c|c|}
\hline \multirow[t]{2}{*}{ Variáveis } & \multicolumn{2}{|c|}{ Modelo 1} & \multicolumn{2}{|c|}{ Modelo 2} & \multicolumn{2}{|c|}{ Modelo 3} \\
\hline & Homem & Mulher & Homem & Mulher & Homem & Mulher \\
\hline Transição do chefe $=0$ & 8,080 & 9,804 & 8,619 & 10,621 & 8,624 & 10,625 \\
\hline Transição do chefe=1 & 15,935 & 11,969 & 17,661 & 14,379 & 17,420 & 14,567 \\
\hline Diferença & 7,855 & 2,165 & 9,042 & 3,757 & 8,796 & 3,942 \\
\hline Variação percentual & 49,295 & 18,085 & 51,198 & 26,131 & 50,496 & 27,063 \\
\hline
\end{tabular}

Fonte: IBGE. Microdados PME 2002 a 2013

Os valores apresentados na Tabela 4, estimados por Oliveira (2005), são inferiores em termos absolutos e relativos aos estimados neste trabalho, apresentando inclusive reversão de sinal. Essas diferenças podem ser decorrentes de atualização dos dados, mudanças em algumas covariáveis, observação da transição dos filhos de 10 a 18 anos e não apenas do filho mais velho e, também, pela incorporação do efeito do plano amostral, que foi ignorado em Oliveira (2005). 
TABELA 4

Estimativas do efeito trabalhador adicional para o filho mais velho

Regiões metropolitanas de Belo Horizonte, Salvador, Porto Alegre, São Paulo, Rio de Janeiro e Recife -1990-2001

\begin{tabular}{|c|c|c|c|c|c|c|}
\hline \multirow{2}{*}{ Variáveis } & \multicolumn{2}{|c|}{ Modelo 1} & \multicolumn{2}{|c|}{ Modelo 2} & \multicolumn{2}{|c|}{ Modelo 3} \\
\hline & Homem & Mulher & Homem & Mulher & Homem & Mulher \\
\hline Transição do chefe=0 & 7,180 & 9,650 & 16,670 & 20,700 & 22,540 & 26,320 \\
\hline Transição do chefe=1 & 8,870 & 7,690 & 18,170 & 10,290 & 24,040 & 22,360 \\
\hline Diferença & 1,690 & $-1,950$ & 1,490 & $-10,420$ & 1,500 & $-3,960$ \\
\hline Variação percentual & 23,538 & $-20,230$ & 8,950 & $-50,320$ & 6,650 & $-15,030$ \\
\hline
\end{tabular}

Fonte: Oliveira (2005).

\section{Considerações finais}

A variável de transição do chefe de família foi estatisticamente significante apenas para os homens, indicando que sua transição para o desemprego aumenta a chance de o filho entrar no mercado de trabalho, evidenciando a existência de um efeito trabalhador adicional positivo, o que não se verificou para as chefes mulheres. A oferta de trabalho dos filhos, para compensar a perda de renda do chefe da família, pode antecipar a entrada de adolescentes e jovens no mercado de trabalho. Em termos conjunturais, o aumento de indivíduos procurando trabalho, no primeiro momento, eleva a taxa de desemprego da economia.

No presente estudo foi considerado qualquer filho, entre 10 e 18 anos, que transitasse para o mercado de trabalho, não se prendendo apenas à transição do filho mais velho, a exemplo de Oliveira (2005).

Não se pode corroborar a hipótese da existência de um efeito trabalhador adicional maior para chefes mulheres, pois, em todos os modelos estimados, a diferença a favor das mulheres foi irrisória. Em que pese a chefia de domicílios feminina estar associada à pobreza, a transição do filho para o mercado de trabalho não parece apresentar seletividade pelo sexo do chefe.

Entretanto, algumas modificações ainda podem ser adicionadas ao modelo, tais como considerar a hipótese de que a oferta de trabalho adicional por parte da família ocorra não somente em função do desemprego do chefe, mas principalmente em virtude da queda da renda familiar. Como, para a média da população brasileira, a transição para a inatividade está relacionada à queda de renda, esta transição pode ser incorporada na variável de transição do chefe para o desemprego.

As transições entre as situações na força de trabalho são eventos dinâmicos, ou seja, a todo o momento existem fluxos de trabalhadores transitando entre ocupação, desemprego e inatividade. Dessa forma, a fixação de apenas um ou dois pontos de transição do chefe para o desemprego ou outra situação na força de trabalho impede que se capte o efeito proveniente de todas as transições. Assim, dever-se-ia fixar apenas o ponto inicial de observação e considerar as transições em todos os demais pontos dos painéis. 
Além do desenvolvimento metodológico, estudos dessa natureza podem contribuir para o planejamento de políticas públicas de educação e primeiro emprego, uma vez que possibilitam incorporar outras variáveis de características familiares e individuais que podem ter poder preditivo da transição compulsória de jovens para o mercado de trabalho.

\section{Referências}

ALTAVILLA, C. et al. Is the discouraged worker effect time varying? Italy: University of Salerno, 2005 (CELPE Discussion Paper, 97).

BENATI, L. Some empirical evidence on the 'discouraged worker' effect. Economics Letters, v. 70, n. 3, p. 387-395, 2001. Disponivel em: 〈http://www.sciencedirect.com/science/article/pii/ S016517650000375X>. Acesso em: 5 jan. 2014.

CONGREGADO, E. et al. Exploring the big jump in the Spanish unemployment rate: evidence on an ‘added-worker' effect. Economic Modelling, n. 28, p. 1099-1105, 2011. Disponível em: «www.elsevi e r.com/locate/ecmod〉. Acesso em: 5 jan. 2014.

CORSEUIL, C. et al. Decisões críticas em idades críticas: a escolha de jovens entre estudo e trabalho em seis países da América Latina. In: ENCONTRO NACIONAL DE ESTUDOS POPULACIONAIS, 12. Brasil 500 anos: mudanças e continuidades. Belo Horizonte: Abep, 2000.

DARBY, et al. Labour force participation and the business cycle: a comparative analysis of France, Japan, Sweden and the United States. Japan and the World Economy, n. 13, p. 113-133, 2001.

DORIS, A. et al. Means testing disincentives and the labour supply of the wives of unemployed men: results from a fixed effects model. Maynooth: National University of Ireland, 1999. Disponivel em: 〈http://www.nuim.ie/academic/economics/pdf/N930999.pdf〉. Acesso em: 30 maio 2004.

DURYEA, S.; LAM, D.; LEVISON, D. Effects of economic shocks on children's employment and schooling in Brazil. Ann Arbor: University of Michigan, Population Study Center, 2003 (PSC Research Report, n. 03-541). Disponivel em: 〈http://www.psc.isr.umich.edu/pubs/pdf/rr03-541.pdf〉. Acesso em: 30 maio 2004.

EHRENBERG, R. G.; SMITH, R. S. A moderna economia do trabalho: teoria e política pública. 5. ed. São Paulo: Makron Books, 2000.

FERNANDES, R. Estratégias de sobrevivência do trabalhador desempregado. In: CHAHAD, J. P. Z.; PICCHETTI, P. A. (Orgs.). Mercado de trabalho no Brasil: padrões de comportamento e transformaç̧̃es institucionais. São Paulo: Ltr, 2003, p. 27-56.

FERNANDES, R.; FELÍCIO, F. O ingresso de esposas na força de trabalho como resposta ao desemprego dos maridos: uma avaliação para o Brasil metropolitano. In: CHAHAD, J. P. Z.; MENEZES-FILHO, N. A. (Orgs.). Mercado de trabalho no Brasil: salário, emprego e desemprego numa era de grandes mudanças. São Paulo: Ltr, 2002, p. 79-96.

GRUBER, J.; CULLEN, J. B. Spousal labor supply as insurance: does unemployment insurance crowd out the added worker effect? Cambridge, MA.: NBER, 1996 (Working paper, n. 5608). Disponivel em: 〈http://www.nber.org/papers/w5608.pdf 〉. Acesso em: 30 maio 2004.

IBGE. Pesquisa Mensal de Emprego: 2002-2013. Rio de Janeiro: IBGE. Disponível em: <ftp://ftp.ibge. gov.br/Trabalho_e_Rendimento/Pesquisa_Mensal_de_Emprego>. Acesso em: 15 nov. 2013.

Série relatórios metodológicos do IBGE: Pesquisa Mensal de Emprego. Rio de Janeiro: IBGE, 2002. Disponível em: 〈ftp://ftp.ibge.gov.br/Trabalho_e_Rendimento/Pesquisa_Mensal_de_Emprego〉. Acesso em: 15 nov. 2013. 
JOTOBÁ, J. A família na força de trabalho: Brasil metropolitano - 1978-1986. In: ENCONTRO BRASILEIRO DE ESTUDOS POPULACIONAIS, 7. Anais... Campinas: Abep, 1990, p.147-174. Disponivel em: 〈http://www. abep.org.br>. Acesso em: 15 dez. 2004.

KASSOUF, A. L. Trabalho infantil: escolaridade x emprego. In: ENCONTRO NACIONAL DE ECONOMIA, 28. Anais... Campinas: Anpec, 2000. Disponivel em CD-ROM.

LEME, M. C. S.; WAJNMAN, S. A alocação do tempo dos adolescentes brasileiros entre o trabalho e a escola. In: ENCONTRO NACIONAL DE ESTUDOS POPULACIONAIS, 12. Brasil 500 anos: mudanças e continuidades. Belo Horizonte: Abep, 2000. Disponível em CD-ROM.

OLIVEIRA, E. L. Transições: três aplicações a partir de dados das pesquisas domiciliares no Brasil. Tese (Doutorado em Demografia). Belo Horizonte: Centro de Desenvolvimento e Planejamento Regional Cedeplar/Universidade Federal de Minas Gerais - UFMG, 2005.

PESSOA, D. G. C.; SILVA, P. L. N. Análise de dados amostrais complexos. Rio de Janeiro, março de 1998. Disponível em: 〈http://www.ie.ufrj.br/download/livro.pdf〉. Acesso em: 5 jan. 2014.

PRIETO-RODRÍGUEZ, J.; RODRÍGUEZ-GUTIÉRREZ, C. Participation of married women in the labour market and the 'added worker effect' in Europe. Luxembourg: IRISS, 2000 (Working paper, n. 12). Disponível em: 〈http://www.ceps.lu/iriss/documents/irisswp15.pdf 〉. Acesso em: 30 maio 2004.

SCHMITT, C.; RIBEIRO, E. P. Participação feminina na força de trabalho e o efeito trabalhador adicional em Porto Alegre. 2003. Disponivel em: 〈http://www.ufrgs.br/ppge/textos-para-discussao. asp?ano=2003>. Acesso em: 28 maio 2004.

SPLETZER, J. R. Reexamining the added worker effect. 1995. Mimeografado.

STEPHENS J. R. M. Worker displacement and the added worker. Cambridge, MA.: NBER, 2001 (Working papers, n. 8260). Disponível em: 〈http://www.nber.org/papers/w8260〉. Acesso em: 28 maio 2004.

\section{Autores}

Elzira Lúcia de Oliveira é doutora em Demografia pelo Centro de Planejamento Regional Cedeplar, da Universidade Federal de Minas Gerais - UFMG. Professora da Universidade Federal Fluminense - UFF Campos.

Eduardo Gonçalves Rios-Neto é PhD em Demografia Econômica pela University of California at Berkeley, EUA. Professor do Centro de Planejamento Regional - Cedeplar, da Universidade Federal de Minas Gerais - UFMG.

Ana Maria Hermeto Camilo de Oliveira é doutora em Demografia pelo Centro de Planejamento Regional - Cedeplar, da Universidade Federal de Minas Gerais - UFMG. Professora do Centro de Planejamento Regional - Cedeplar, da Universidade Federal de Minas Gerais - UFMG.

\section{Endereço de correspondência}

Elzira Lúcia de Oliveira

Av. Atlantica, 1324

Chapéu de São João da Barra, RJ - CEP 28200-000 


\begin{abstract}
The added worker effect for children in Brazil

The aim of this paper was to test the hypothesis of the existence of the effect of the added worker for children in Brazil. The paper aims to identify whether a situation of unemployment of the head of the household will lead some daughter or son in the family to enter the economically active population (EAP). The database used was the Monthly Employment Survey conducted by IBGE, which allows the construction of panels for longitudinal data analysis. The hypothesis was tested for at least one child aged 10 to 18, between 2002 and 2013, for the metropolitan areas of Belo Horizonte, Salvador, Porto Alegre, Sao Paulo, Rio de Janeiro and Recife, which make up the area covered by the Monthly Employment Survey (PME). This study accepts the existence of differential by gender of the head of the household for the added worker effect for children ages 10-18. The results showed that there was a greater positive effect for male heads of household than for female heads of household. The variable of transition of the son or daughter to employed activity showed no statistical significance that would allow us to assume the existence of the effect. The hypothesis of the existence of a differential by gender was confirmed, but in the opposite direction from the original hypothesis.
\end{abstract}

Keywords: Additional worker effect. Unemployment. Labor market.

\title{
Resumen
}

El efecto trabajador adicional para hijos en Brasil

El objetivo de este artículo es probar la hipótesis de la existencia del efecto trabajador adicional para hijos en Brasil, tratando de identificar si la situación de desempleo del jefe de familia hará que algún miembro de la familia, cuya condición sea de hijo, transite hacia la Población Económicamente Activa - PEA. La base de datos utilizada fue la Pesquisa Mensal de Emprego - PME, realizada por IBGE, que permite la construcción de paneles para el análisis longitudinal de datos. La hipótesis fue probada para por lo menos un hijo con edad de 10 a 18 años, entre 2002 y 2013, para las regiones metropolitanas de Belo Horizonte, Salvador, Porto Alegre, São Paulo, Rio de Janeiro y Recife, que conforman el área de alcance de la PME. Este trabajo admite la existencia de diferencial por sexo del jefe para el efecto trabajador adicional de hijos de 10 a 18 anos. Los resultados mostraron que hay un efecto positivo mayor para jefes hombres que para jefas mujeres, siendo que la variable de transición del hijo hacia la actividad no presentó significancia estadística que permitiera asumir la existencia del efecto. Se corroboró la hipótesis de la existencia de diferencial por sexo, pero en el sentido opuesto al de la hipótesis asumida.

Palabras-claves: Efecto trabajador adicional. Desempleo. Mercado de trabajo.

Recebido para publicação em 27/07/2011 Aceito para publicação em 30/10/2011 
